\title{
Teaching New Mothers about Infant Feeding Cues May Increase Breastfeeding Duration
}

\author{
Jay Kandiah, Charlene Burian, Valerie Amend
}

Department of Family and Consumer Sciences, Ball State University, Muncie, USA.

Email: vaamend@bsu.edu

Received January $20^{\text {th }}, 2011$; revised February $17^{\text {th }}, 2011$; accepted February $24^{\text {th }}, 2011$.

\begin{abstract}
The objective of this pilot study was to compare two different methods of educating prenatal women regarding breastfeeding. Comparisons were made between traditional and innovative methods to determine which was more effective in increasing breastfeeding duration. Over a 32 month period, 197 prenatal women were assigned to either a control $(C, n$ $=139)$ or an experimental $(E, n=51)$ group. The $C$ group received standard breastfeeding education, while the $E$ group received standard education in addition to information about infant hunger cues. Cox Regression and KaplanMeier analysis were performed. Estimated mean number of weeks for $C$ and E groups to continue breastfeeding was $14.3+17.4$ weeks and $18.5+17.1$ weeks, respectively. At 26 weeks, duration of breastfeeding approached significance (chi square $=2.907, d f=1, p=0.088$ ), indicating probability of continuing to breastfeed was about $28 \%$ better for those in E group when compared to $C$ group. Duration of breastfeeding may increase when prenatal women are taught to identify infant behavior such as hunger cues.
\end{abstract}

Keywords: Breastfeeding Duration, Infant Feeding Cues, WIC

\section{Introduction}

The American Academy of Pediatrics has recommended that the optimal way to feed an infant is to breastfeed for the first year of life [1]. Similar recommendations have been made by the American Dietetic Association [2] and the World Health Organization [3]. In spite of recommendations and supporting evidence proclaimed by credible organizations, formula feeding continues to be prevalent in the United States. An objective of Healthy People 2010 was to increase the proportion of new mothers initiating breastfeeding of infants from $64 \%$ to $75 \%$ [4]. Additionally, it has been recommended that exclusive breastfeeding during early post-partum be increased from $29 \%$ (1998) to 50\% [4]. Hurley et al., found between 2004 and 2006, 67\% of mothers participating in the Women, Infant and Children Special Supplemental Feeding Program (WIC) initiated breastfeeding. Although initiation of breastfeeding approached the national goal of $75 \%$, the rate at 6 months was $21 \%$, which was far below the desired Healthy People 2010 goal [5].

The WIC Program serves low-income prenatal women and their infants, and since August 1985, has spearheaded breastfeeding promotion campaigns locally and nationwide [6]. Some of the responsibilities of the WIC program include explaining the benefits of breastfeeding, providing informational and educational sessions, and provision of breast pumps.

Exclusive breastfeeding for 6 months or more has been linked to many health benefits including lower incidence of obesity, diabetes, childhood anemia, and dental problems. Most studies have looked at health and social benefits of breastfeeding. Overwhelming medical evidence related to breastfeeding include a significant decrease in pediatric healthcare costs, decreased infant/ child morbidity and mortality, protection against common pediatric infections and chronic diseases [3,4,7]. In addition, many researchers have looked at duration of breastfeeding and/or the physiological benefits of breastfeeding [8-10]. However, few if any studies have compared the effectiveness of various teaching techniques to promote duration of breastfeeding.

Statistics for WIC reveal that many mothers are initiating breastfeeding while in the hospital, but the number dwindles off dramatically after the first month of post partum. Ross Mother's Survey [11] reported that 56.8\% of WIC mothers initiated breastfeeding in the hospital, but only $20.1 \%$ reported continuation of breastfeeding at 6 months. Schwartz, et al., concluded that although participation in WIC programs helped in-crease initiation of 
breastfeeding, the advice clients received from WIC did not impact duration of breastfeeding [12]. Therefore, it is logical to assume that the largest stumbling block to breastfeeding with this population is not so much a lack of information about the benefits of breastfeeding but investigating effective strategies to increase breastfeeding duration.

\section{Review of the Literature}

The 2005 recommendations by the International Lactation Consultant Association's Clinical Guidelines for the Establishment of Exclusive Breastfeeding has made suggestions to the medical community to "Teach mothers to recognize and respond to early infant feeding cues and confirm that the baby is being fed at least 8 times in each 24 hours" [13]. Some of the components discussed were watching infant feeding cues such as sucking and handto-mouth movements. Chiu, Lau, Kuo, and Chung [14] conducted a study with 60 second year nursing students, to explore avenues as to how they would instruct post partum patients on observing baby cues related to hunger or satiety. Only $26 \%(n=16)$ of the nurses were able to identify hunger cues, among other infant behaviors. Since many WIC clients are young mothers with fragile support systems available for instructing them about parenting, these findings demonstrated that nurses have been insufficiently trained in teaching their clients about infant behavior.

Bonuck, Trombley, Freeman, and McKee [15] compared the difference between the use of standard care or prenatal and post natal care with an International Board Certified Lactation Consultant (IBCLC) in evaluating breastfeeding intensity in 304 participants. Using telephone interviews the randomized, unmasked, controlled prospective trial design investigated feeding patterns of infants from birth to 12 months. The researchers found when compared to the control, the intervention group was more likely $(p=0.028)$ to breastfeed up to 20 weeks post-partum. Similarly, Lukac, Riley and Humphry [16] used a retrospective chart review to examine IBCLC's effectiveness on increasing duration of breast-feeding among new mothers and newborns. The re-searchers compared breastfeeding rates at 4 - 6 months for mothers in the control and the experimental groups. The experimental group received counseling from an IBCLC while the control group received advice from a nutrition-ist who was not an IBCLC. Results indicated $53 \%$ of mothers seen by the IBCLC were still breastfeeding at $4-6$ months, compared to $23 \%$ of the control group. LawlerSmith, McIntyre, and Bruce [17] used a 12-month preand post-intervention patient completed survey to obtain data to determine the value of an IBCLC led breastfeeding intervention program on duration of breastfeeding.
Results indicated that at 24 and 26 weeks postpartum, the rates for solely breastfeeding were significantly higher in the intervention group ( $p=0.015$ and $p=0.018$, respectively).

Although numerous studies have suggested a positive correlation between IBCLC use and breastfeeding duration, few studies have explored the expertise of IBCLC along with the efficacy of various teaching strategies designed to improve breastfeeding duration. Thus the present research was conducted to further examine specific strategies designed to increase the duration of breastfeeding among low-income women, particularly in a federally funded program, Women Infant and Children's Special Supplemental Feeding Program. Previously, investigators have included a partner-supported, incentivebased program that looked at breastfeeding initiation rates along with duration rates [18] or pre- and post-natal counseling by trained IBCLC's [19]. However, to date, no research has investigated using different educational tools and the involvement of a registered dietitian (RD) or an RD with IBCLC's (RD + IBCLC) role in increasing breastfeeding duration. In order to enable new mothers to continue to breastfeed for a longer duration of time, the most effective strategies need to be identified.

Therefore, this pilot study was conducted to compare two different methods of educating prenatal women regarding breastfeeding in consultation with either an $\mathrm{RD}$ or an RD + IBCLC. Comparisons were made between the traditional method (i.e. stating the benefits of breastfeeding in consultation with an RD) and a different method (i.e. discussing infant feeding cues and behavior with an RD + IBCLC) to determine which method was more effective in increasing breastfeeding duration, and when breastfeeding would cease. The hypothesis of this study was that discussing infant feeding cues and behaviors with a RD + IBCLC will increase breastfeeding duration of mothers.

\section{Methods}

This research relates to nutrition and wellness in regards to nutritional status of populations and practices to increase consumer nutrition knowledge. In particular, this manuscript will discuss two types of strategies utilized by an RD or an RD + IBCLC in promoting breastfeeding duration among low-income WIC mothers. The mission of the WIC program is to safeguard the health of lowincome women, infants, and children up to age five who are at nutrition risk by providing nutritious foods to supplement diets, information on healthy eating, and referrals to health care [6]. IBCLC's are all either Registered Nurses or RDs. All WIC RD's and IBCLC's attend a three day nutrition counseling course that addresses the importance of breastfeeding to both the mother and baby. 
Prior to the start of the research, and after approval by the University's Institutional Committee on Investigations Involving Human Subjects, 200 prenatal women (18 years and older) at a Midwestern WIC clinic who met the federal guidelines volunteered to participate. To be qualified into the WIC program, participants must have a household income of less than $185 \%$ of the federally determined poverty level and should have some nutritional risk criteria [6]. Upon admittance into the WIC program, clients were certified by an RD. After approval of the client's signed consent each participant was assigned to either a control group (C) under the supervision of an $\mathrm{RD}$ or an experimental (E) group depending on availability of the RD + IBCLC. The C group received the standard education related to the benefits of breastfeeding through brochures entitled " 20 Great Reasons to Breastfeed" and "Is Breastfeeding Right for Me?" This information was provided to them by an RD. The E group received the standard education similar to the control group along with information related to infant feeding cues and behaviors. The E group also received a flyer describing infant feeding cues, "How to Tell if Your Baby is Hungry", a hand out describing an infant's second night at home and a tear off handout entitled "Breastfeeding: A Special Relationship" that described and discussed how to hold a baby for feedings and latching. Unlike the control group, this information was provided to participants through the RD + IBCLC.

As a mandate to the WIC program, all certified prenatal clients had to visit a registered dietitian at least twice prior to their delivery. Although both groups visited the RD twice, subjects in the E group had an additional one-time 30 minute visit with a RD + IBCLC to discuss in-depth topics such as normal infant behaviors, infant feeding cues and behavioral patterns of an infant. During this time, participants were also given an opportunity to ask any questions or report any concerns they had regarding breastfeeding and infant feeding cues.

After post-partum, the $\mathrm{C}$ and $\mathrm{E}$ groups returned to the WIC clinic to be recertified, where their records were updated and breastfeeding data was collected. Information regarding breastfeeding duration was collected at three month intervals, and comparisons were made between the groups. Data was collected by means of WIC postpartum certification. At the first visit of the postpartum mother to WIC clinic for recertification, she was certified either as breastfeeding or non-breastfeeding. In addition, since WIC is a federally funded program one of the requirements is to gather a record of breastfeeding status (i.e. initiation of breastfeeding, duration of breastfeeding, rational for ceasing breastfeeding, and if breastfeeding was supple-mented with infant formula).

A Cox proportional-hazards regression analyzed the influence of standard education with an $\mathrm{RD}$ and infant feeding cues with RD + IBCLC on breastfeeding duration. The data was also analyzed using the Mann-Whitney test and Kaplan-Meier procedure. The Cox Regression analysis and the Kaplan-Meier were used because they are suited for situations when duration is of interest but not all cases have reached conclusion. These statistics are preferred in medical research in judging the efficacy of a treatment in situations where cases would otherwise be deleted because of missing outcome data, such as ordinary least squares regression [20] In the current study, participants could be breastfeeding longer than the collection of breastfeeding data ( 24 months or 52 weeks).

\section{Results}

The research was over a 32 month period. Breastfeeding data for each participant was collected for 24 months. Since there was variability among participants as to the time of gestation and when they were certified into WIC, data collection began in October 2002 and ended in June 2005. The objective of this was to include participants who continued to breastfeed until they were no longer eligible for WIC.

From a pool of 200 participants, 190 pre- and postpartum mothers successfully completed the research $(\mathrm{C}=$ $139, \mathrm{E}=51)$. The sample in the $\mathrm{E}$ group was smaller because it was based on the work schedule of the RD + IBCLC and her other commitments. As observed in Table 1, majority of the participants were between $18-23$ years of age and were predominately Caucasians. The two groups were very similar in age and ethnicity. The demographical information is gathered when a person applies for the WIC program during the initial applications process. Criteria into WIC are based on financial eligibility (all participants income was $\leq 180 \%$ of the federally mandated poverty line), and the application is processed by a clerical staff in the WIC program, not by an RD or RD + IBCLC. In reference to ethnicity, the population is a mid-western, mid-sized heterogeneous mixture of predominantly Caucasians $(83.7 \%)$, followed by African-Americans (11.9\%), then Hispanics (6.4\%).

Table 1. Participants demographic information $(n=190)$.

\begin{tabular}{clc}
\hline Characteristics & Description & Number \\
\hline \multirow{3}{*}{ Age (years) } & $18-23$ & 92 \\
& $24-29$ & 66 \\
& $\geq 30$ & 32 \\
& Caucasian/White & 85 \\
& African American & 49 \\
\multirow{5}{*}{ Ethnicity } & Asian/Pacific Islander & 0 \\
& Hispanic/Latino & 56 \\
& Other & 0 \\
\hline
\end{tabular}


Since the purpose of the research was to investigate if there was a difference in the duration of breastfeeding and when breastfeeding ceased in the $\mathrm{C}$ and $\mathrm{E}$ groups, a Cox Regression and a Kaplan-Meier analysis were performed. These analyses were used to predict a trend that might occur for data that does not have a definitive end or outcome, also known as censored data. In this case, participants may be breastfeeding longer than one year (52 weeks) when they would no longer be eligible for WIC. The records however would only reflect that the participant had not stopped breastfeeding as of 52 weeks, and the total number of weeks the client may have breastfed beyond that time would be unknown.

A Cox Regression analysis of the 190 participants at the end of 52 weeks using standard education and infant feeding cues as a predicator of duration of breastfeeding was found not to be statistically significant $\left(\chi^{2}=1.548\right.$, $d f=1, p=0.213$ ). As such, the Kaplan-Meier test was performed to examine potential trends that may be occurring between the $\mathrm{C}$ and $\mathrm{E}$ groups. Figure 1, shows there appears to be some separation between the two groups up to 30 weeks with the E group having a stronger probability of continuing breastfeeding behaviors. As shown in Table 2, estimated mean number of weeks for the $\mathrm{C}$ and $\mathrm{E}$ groups appears to be $14.3 \pm 17.4$ and $18.5 \pm$ 17.1 weeks respectively.

Since the separation between the $\mathrm{C}$ and $\mathrm{E}$ groups occurred at 30 weeks, further analysis was conducted to identify if there was any variation at 6 months (i.e. at 26

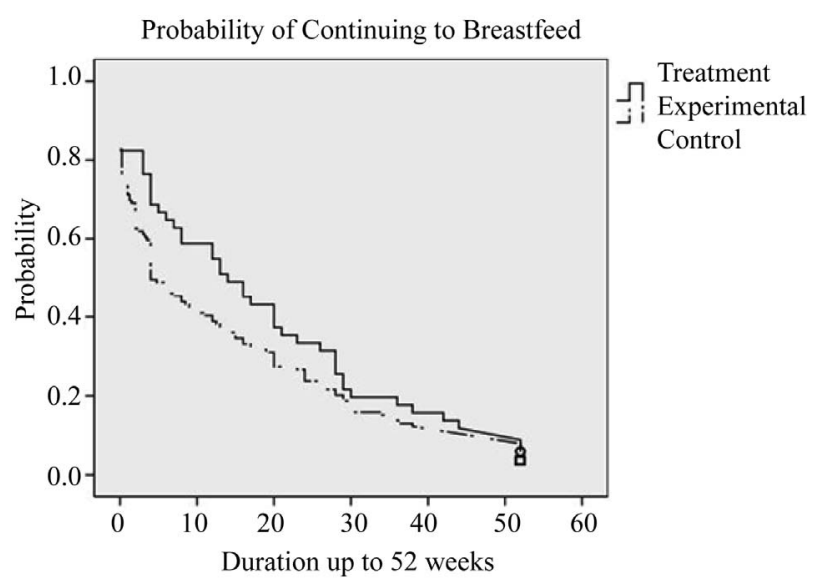

Figure 1. Potential trends in breastfeeding duration between $\mathrm{C}$ and $\mathrm{E}$ groups -52 weeks.

Table 2. Estimated mean ( \pm SD) number of week’s par ticipants continued breast feeding.

\begin{tabular}{ccc}
\hline Group & 26 weeks & 52 weeks \\
\hline Control (C) & $10.6 \pm 10.6$ & $14.3 \pm 17.4$ \\
Experimental (E) & $14.1 \pm 10.4$ & $18.5 \pm 17.1$ \\
\hline
\end{tabular}

weeks). Cox regression analysis of the 190 participants at 26 weeks using standard education along with infant feeding cues as a predicator of duration of breastfeeding approached significance $\left(\chi^{2}=2.907, d f=1, p=0.088\right)$. The estimated treatment mean was 268 indicating a $27 \%$ improvement in the probability of continuing to breastfeed. As observed in Figure 2, the Kaplan-Meier test shows how the probability of continuing to breastfeed varies across the observed period. As shown in Table 2, estimated mean number of weeks for the $\mathrm{C}$ and $\mathrm{E}$ groups to continue breastfeeding was $10.6 \pm 10.6$ and $14.1 \pm$ 10.4 weeks respectively.

\section{Discussion and Conclusions}

Unlike previously mentioned studies, to the best of the investigators' knowledge, to date, no research has compared two types of strategies utilized by an RD or an $\mathrm{RD}+\mathrm{IBCLC}$ in promoting breastfeeding duration among low-income WIC mothers. Therefore, this pilot study was conducted to compare between the standard methods (education related to the benefits of breastfeeding through brochures) with an RD and a different method (the standard method along with information related to infant feeding cues and behaviors) with an RD + IBCLC. The hypothesis of this study was that discussing infant feeding cues and behaviors with a RD + IBCLC will increase breastfeeding duration of mothers.

To reiterate the findings of the current pilot study, pregnant women who received information about infant feeding cues along with the standard education about breastfeeding (E group) tended to breastfeed for a longer duration than those who received only the standard education (C group). It was also noted that at 52 weeks there was no statistical significant difference in breastfeeding duration between the two groups. However, when compared to the $\mathrm{C}$ group, duration of breastfeeding appro-

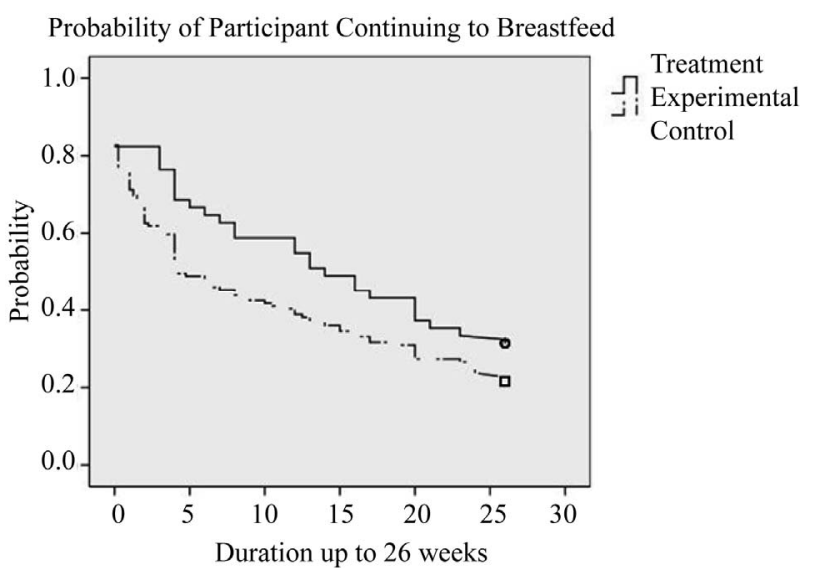

Figure 2. Potential trends in breastfeeding duration between $\mathrm{C}$ and $\mathrm{E}$ groups -26 weeks. 
ached significance in the E group indicating the potential trend to continue to breastfeed after the duration of the study was $27 \%$ overall better for the E group. Although the study demonstrated duration of breastfeeding increased when infant feeding cues and behaviors with an RD + IBCLC was utilized, it was not statistically significant between the $\mathrm{E}$ and $\mathrm{C}$ groups. Statistical power was not observed in the E group due to the small sample size.

This research is unique in that it addressed different types of teaching methods, specifically the influence of infant feeding cues on duration of breastfeeding and involvement of RD + IBCLC. Previous researchers have looked at various benefits of breastfeeding to the mother and infant including the physiological benefits of breastfeeding and breast milk [21,22] and the negative impact of maternal employment and family support [23,24]. Unlike other studies, in this research, the control and experimental groups were of similar socio-economic status and geographical location. The population was a heterogeneous mixture of Caucasian, African-Americans, and Hispanics, and as such, results could be generalized. During the entire study period, clients were seen, counseled, and received breastfeeding education from the same RD and RD + IBCLC from one WIC clinic.

This research was limited to WIC participants from one county, and as such, results may vary within other areas. A possible interfering factor was the availability of infant formula at the WIC office and hospitals where the babies were delivered. Numerous researchers have demonstrated the interference of infant formula supplemental feedings with the duration of breastfeeding. Howard et al., [25] conducted a randomized clinical trial demonstrating the negative effects of not only supplemental feedings, but pacifier use as a hindrance to breastfeeding. Similarly Chezem et al., [23] found that hospital use of formula packs had a negative effect on breastfeeding duration, especially among women planning postpartum employment. It could be speculated if participants in this study had not received formula feeding, they may have resorted to breastfeeding. This could have facilitated mothers to have a better understanding of infant feeding cues, thereby possibly increasing duration of breastfeeding.

Recommendations for future research include: 1) a larger sample from different geographical locations; 2) equal distribution of control and experimental groups; 3 ) development and comparison of innovative educational resources that may increase breastfeeding duration; and 4) investigation of maternal, spousal, and extended families role in contributing to mother's duration of breastfeeding and understanding of infant feeding cues. This pilot study demonstrates the possible efficacy of utilizing different strategies with an RD + IBCLC to identify infant feeding to increase breastfeeding duration. Therefore, there may be a benefit of such strategies as a component of WIC's educational program.

\section{REFERENCES}

[1] American Academy of Pediatrics, "Poicy Statement: Breastfeeding and the Use of Human Milk," Pediatrics, Vol. 115, No. 2, 2005, pp. 496-506. doi:10.1542/peds.2004-2491

[2] American Dietetic Association, "Position of the American Dietetic Association: Promoting and Supporting Breastfeeding," Journal of the American Dietetic Association, Vol. 105, No. 5, 2009, pp. 810-818.

[3] World Health Organization, "Global Strategy for Infant and Young Child Feeding," World Health Organization, Geneva, 2003.

[4] United States Department of Health and Human Services, "Healthy People 2010: Conference Edition: Volumes I and II," US Department of Health and Human Services, Washington DC, 2010.

[5] K. Hurley, et al., "Variation in Breastfeeding Behaviours, Perceptions, and Experiences by Race Ethnicity among a Low-Income Statewide Sample of Special Supplemental Nutrition Program for Women, Infants, and Children (WIC) Partipants in the United States," Maternal and Child Nutrition, Vol. 4, No. 2, 2008, pp. 95-105. doi:10.1111/j.1740-8709.2007.00105.x

[6] United States Department of Agriculture, "Women, Infants, and Children," 2010. http://www.fns.usda.gov/wic/

[7] J. Weimer, "The Economical Cost of Breastfeeding: A Review and an Analysis," US Department of Agriculture, Washington DC, 2001.

[8] G. Balaban, et al., "Early Weaning and Other Potential Risk Factors for Overweight among Preschool Children," Clinics, Vol. 65, No. 2, 2010, pp. 181-187. doi:10.1590/S1807-59322010000200010

[9] M. S. Kramer, et al., "Health and Development Outcomes in $6.5 \mathrm{yr}$-Old Children Breastfed Exclusively for 3 to 6 Months," American Journal of Clinical Nutrition, Vol. 90, No. 4, 2009, pp. 1070-1074. doi:10.3945/ajcn.2009.28021

[10] A. M. Stuebe and E. B. Schwartz, "The Risks and Benefits of Infant Feeding Practices for Women and Their Children," Journal of Perinatology, Vol. 30, No. 3, 2010, pp. 155-162. doi:10.1038/jp.2009.107

[11] Ross Products Division, Abbott Laboratories, "The Mothers Survey," Ross Products Division, Abbott Laboratories, 2000, pp. 1-12.

[12] B. J. Schwartz, et al., "Does WIC Participation Improve Breastfeeding Practices?" American Journal of Public Health, Vol. 85, No. 5, 2005, pp. 727-731.

[13] M. L. Overfield, et al., "Clinical Guidelines for the Establishment of Exclusive Breastfeeding," 2005. http://www.ilca.org/files/resources/ClinicalGuidelines200 5.pdf

[14] F. U. Chiu, et al., "Common Problems of Clinical Performance Examination in Breastfeeding Instruction for Nurs- 
ing Baccalaureate Students," Journal of Nursing Resources, Vol. 11, No. 2, 2003, pp. 109-118. doi:10.1097/01.JNR.0000347626.57229.3e

[15] K. A. Bonuck, et al., "Randomized, Controlled, Trial of Prenatal and Post-Natal Lactation Cosultant on Duration and Intensity of Breastfeeding up to 12 Months," Pediatrics, Vol. 116, No. 6, 2005, pp. 1413-1426. doi:10.1542/peds.2005-0435

[16] M. Lucak, et al., "How to Integrate a Lactation Consultant in an Outpatient Clinic Environment," Journal of Human Lactation, Vol. 22, No. 1, 2006, pp. 99-103.

[17] C. Lawler-Smith, et al., "Effective Breastfeeding Support in a General Practice," Australian Family Physician, Vol. 26, No. 5, 1997, pp. 573-575 and 578-580.

[18] J. P. Sciacca, et al., "Influences on Breastfeeding by Lower Income Women: An Incentive-Based, Partner-Supported Educational Program," Journal of American Dietetic Association, Vol. 95, No. 3, 1995, pp. 323-328.

[19] N. B. Brent, et al., "Breastfeeding in a Low Income Population: Program to Increase Incidence and Duration," Archives of Pediatric and Adolescent Medicine, Vol. 149, No. 7, 1995, pp. 798-803.
[20] T. Martinussen and T. H. Scheike, "Dynamic Regression Models for Survival Data," Springer, New York, 2006.

[21] R. Meyer, "Infant Feed First Year. 1: Feeding Practices in the First Six Months of Life," Journal of Family Health Care, Vol. 19, No. 1, 2009, pp. 13-16.

[22] T. C. Wu and P. H. Chen, "Health Conesquences of Nutrition in Childhood and Early Infancy," Pediatric Neonatology, Vol. 50, No. 4, 2009, pp. 135-142.

[23] J. C. Chezem, et al., "Lactation Duration: Influences of Human Milk Replacements and Formula Samples in Women Planning Postpartum Employment," Journal of $\mathrm{Ob}$ stetrics and Gynecological and Neonatal Nursing, Vol. 27, No. 6, 1998, pp. 646-651. doi:10.1111/j.1552-6909.1998.tb02634.x

[24] D. Wade, et al., "Breastfeeding Peer Support: Are There Additional Benefits?" Journal of Community Practices, Vol. 82, No. 12, 2009, pp. 30-33.

[25] C. R. Howard, et al., "Randomized Clinical Trial of Pacifier Use and Bottle Feeding or Cup Feeding and Their Effect on Breastfeeding," Pediatrics, Vol. 111, No. 3, 2003, pp. 511-518. 